\title{
Placenta Percreta and Incomplete Uterine Rupture after Endometrial Ablation and Tubal Occlusion
}

\author{
Jaden R. Kohn, BS ${ }^{1}$ Edwina Popek, DO ${ }^{2}$ Concepcion R. Diaz-Arrastia, MD ${ }^{3,4}$ Xiaoming Guan, MD, $\mathrm{PhD}^{3}$
} Alireza A. Shamshirsaz, MD ${ }^{3}$ Michael A. Belfort, MD, PhD ${ }^{3}$ Karin A. Fox, MD, MEd ${ }^{3}$

${ }^{1}$ Baylor College of Medicine, Houston, Texas

2 Department of Pathology, Baylor College of Medicine,

Houston, Texas

3 Division of Maternal-Fetal Medicine, Department of Obstetrics and Gynecology, Baylor College of Medicine, Houston, Texas

${ }^{4}$ Department of Obstetrics, Gynecology and Reproductive Sciences, McGovern Medical School, Houston, Texas
Address for correspondence Karin A. Fox, MD, MEd, Division of Maternal-Fetal Medicine, Department of Obstetrics and Gynecology, Baylor College of Medicine, Texas Children's Pavilion for Women, 6651 Main St, Suite F1020, Houston, TX 77030

(e-mail: Karin.Fox@bcm.edu).

Am J Perinatol Rep 2016;6:e445-e450.

\begin{abstract}
Keywords

- morbidly adherent placenta

- placenta percreta

- bilateral tubal sterilization

- endometrial ablation

- uterine rupture
\end{abstract}

Endometrial ablation offers symptomatic relief for menorrhagia. Pregnancy after ablation is rare but is often complicated due to pregnancy loss, growth restriction, preterm premature rupture of membranes, preterm delivery, and morbidly adherent placentation, a dangerous complication that can result in hemorrhage, intensive care unit admission, and cesarean hysterectomy. We report a case of pregnancy conceived contemporaneously with endometrial ablation and tubal occlusion. Diagnosis of pregnancy was delayed due to low suspicion. Complications included cervical implantation and placenta percreta, necessitating hysterectomy with the fetus in situ. Intraoperatively, incomplete uterine rupture was noted. Abnormal neovascularization, fibrous adhesions, and anatomical distortion necessitated a complex surgical approach. Women undergoing endometrial ablation must be thoroughly counseled about the serious risks of postablation pregnancy, the need for contraception, and the risk of sterilization failure. Pregnancy should remain in the differential diagnosis for women of reproductive age, regardless of tubal occlusion. Cases of placenta percreta should be referred early to centers of excellence with multidisciplinary teams.

Morbidly adherent placentation (MAP), which includes placenta accreta, increta, and percreta, is a serious complication of pregnancy associated with hemorrhage, intensive care unit admission, and cesarean hysterectomy. ${ }^{1}$ MAP complicates approximately 1 in 500 to 1,000 pregnancies $^{1}$; this rate has increased up to 10 -fold in the past 20 years, attributed largely to rising rates of cesarean deliveries. ${ }^{2}$ Additional risk factors for MAP include placenta previa, uterine surgery other than cesarean delivery, advanced maternal age, multiparity, and history of endometrial ablation. ${ }^{3}$

received

October 20, 2016 accepted after revision

November 21, 2016
DOI http://dx.doi.org/

10.1055/s-0036-1597892. ISSN 2157-6998.
It has been estimated that 10 to $30 \%$ of premenopausal and perimenopausal women suffer from abnormal uterine bleeding, which significantly affects quality of life and healthcare costs. ${ }^{4,5}$ Endometrial ablation is an effective therapeutic alternative to hysterectomy in the treatment of menorrhagia and is associated with reduced morbidity, cost, and recovery time. ${ }^{6}$ Ablation destroys the functionalis layer of the endometrium and may result in intrauterine adhesions, atrophy, and fibrosis leading to infertility; however, endometrial ablation is not a contraceptive method. If functional
Copyright $\odot 2016$ by Thieme Medical Publishers, Inc., 333 Seventh Avenue, New York, NY 10001, USA. Tel: +1(212) 584-4662.
License terms

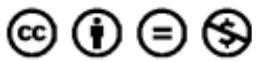


endometrium remains, postablation pregnancy is possible. Depending on the method of ablation, the postprocedure pregnancy rate is approximately 0.24 to $5.2 \%^{7-9}$ These pregnancies are associated with poor outcomes including ectopic pregnancy, spontaneous abortion, intrauterine growth restriction, preterm premature rupture of membranes, preterm delivery, intrauterine fetal demise, uterine rupture, hemorrhage, MAP, and maternal and neonatal mortality. ${ }^{10,11}$

\section{Case}

A 28-year-old G5P2214 woman with three prior cesarean deliveries and one prior dilation and curettage underwent endometrial ablation for menorrhagia refractory to medical management with oral contraceptives using NovaSure (Hologic Inc., Marlborough, MA) with simultaneous laparoscopic bilateral tubal occlusion through Falope-Ring Band (Gyrus ACMI/Olympus, Center Valley, PA). Prior to ablation and tubal occlusion, urine pregnancy test was negative. Eight weeks after surgery, she developed heavy vaginal bleeding with clots and abdominal pain. She thought her ablation had failed and that the pain was from an incisional hernia. She presented to a local emergency room, where point-of-care qualitative urine human chorionic gonadotropin was negative. Computed tomography scan of the abdomen and pelvis showed intrauterine debris. She was discharged home with instructions to return if bleeding or pain recurred.

Ten weeks later, she followed up with the obstetriciangynecologist who had performed her ablation and tubal occlusion. Bleeding had decreased to occasional spotting but she was found to be 18 weeks pregnant (based on fetal biometry on ultrasound), which placed her estimated date of conception on the day of ablation; however, the patient reported that she had been abstinent for 2 weeks following ablation. The placenta was low lying with inability to visualize the cervix, so her obstetrician referred her immediately to our morbidly adherent placenta service for suspected placenta accreta.
Upon arrival to our institution at 20w0d, she underwent transabdominal ultrasound and magnetic resonance imaging (MRI) evaluation. Our protocol relies upon ultrasound as the initial imaging modality for MAP, after which MRI is performed if invasion beyond the uterus is suspected or if the diagnosis remains uncertain. Our MRI protocol includes obtaining images using $6-\mathrm{mm}$ slices at $1.5 \mathrm{~T}$ with a sufficiently wide view to visualize the entire uterus and cervix. In this patient, both the transabdominal ultrasound and the MRI were highly suggestive of placenta percreta with complete obliteration of the cervix.

Transabdominal ultrasound findings (-Fig. 1, Panel A) included complete placenta previa and the inability to visualize the cervix, as well as multiple irregular placental lacunae, bulging of the placenta toward the bladder, and the absence of the retroplacental echolucency, which suggested invasive placentation. Color Doppler imaging demonstrated abnormal vasculature feeding the placenta, as a branch of the left uterine artery appeared to extend directly to the midline of the uterus and placenta (not shown in figures).

On MRI ( - Fig. 1, Panels B and C), the placenta was reported to be entirely inferior, covering the expected location of the cervical os, with several rounded and bandlike areas of low T2 signal, particularly in the posterior placenta. Along the left lateral margin of the lower uterine segment, there were additional areas of bandlike low $\mathrm{T} 2$ signal and an approximately 8 -cm-wide disruption of the myometrium. These bandlike areas of low T2 signal suggest invasive placentation, and a high volume of these areas is suggestive of placenta percreta.

Due to the inability to visualize the cervix on both transabdominal ultrasound and MRI, we planned to perform transvaginal ultrasound, but just prior to this procedure, the patient bled an estimated $250 \mathrm{~mL}$ and was immediately admitted to the antepartum unit. Laboratory studies were as follows: hemoglobin: $10.6 \mathrm{~g} / \mathrm{dL}$; hematocrit: $31.3 \%$; international normalized ratio: 1.0; activated partial thromboplastin time: 28 seconds; fibrinogen: $424 \mathrm{mg} / \mathrm{dL}$. She underwent extensive counseling about the risks of continuing the


Fig. 1 Radiographic imaging. Panel A: transabdominal ultrasound, rotated 90 degrees to align with sagittal magnetic resonance imaging (MRI; Panel B). Asterisk $\left(^{*}\right)$ indicates tortuous, irregular placental lacunae and loss of the hypoechoic zone-BL, bladder; CX, cervical region. Panel B (sagittal) and Panel C (coronal): T2-weighted MRI demonstrating fundus of the uterus with an empty intrauterine cavity (white arrows), fetus visible in the lower uterine segment, and inferior placenta with areas of bandlike low T2 signal (asterisk). Images obtained using 6-mm slices at 1.5T. Note the lack of clear cervical imaging on both MRI and ultrasound. 
pregnancy (uterine rupture, massive hemorrhage, and the potential need to intervene with emergent surgery) versus the outcome of pregnancy loss with immediate treatment. She remained stable with continued spotting overnight, and after consultation with her family, she consented the next morning to cesarean hysterectomy.

Our multidisciplinary percreta team performed a hysterectomy with the fetus in situ at 20 weeks and 1 day. Upon opening the abdomen, we observed that the fundus of the uterus was not distended and appeared distinct from a markedly widened and hypervascular lower uterine segment, which contained the anterior placental edge ( - Fig. 2, Panel $\mathrm{G})$. Multiple, large maternal vessels entered the placenta along the anterior wall of the uterus, and thick fibrous adhesions connected the uterus to the posterior bladder wall.

At the superior margin of the distended lower uterine segment, we observed two large regions of partial uterine dehiscence, defined as a disruption of the myometrium covered by intact but transparent serosa, referred to anecdotally as a "uterine window" (-Fig. 2, Panels B-E). These regions of partial dehiscence permitted clear visualization of the fetus ( - Fig. 2, Panel C) and were concerning for impending uterine rupture. The placental tissue caused a bulge in the weakened left side of the lower uterine segment, while additional placental tissue stretched and angled the lower uterine segment and cervix toward the right pelvic sidewall
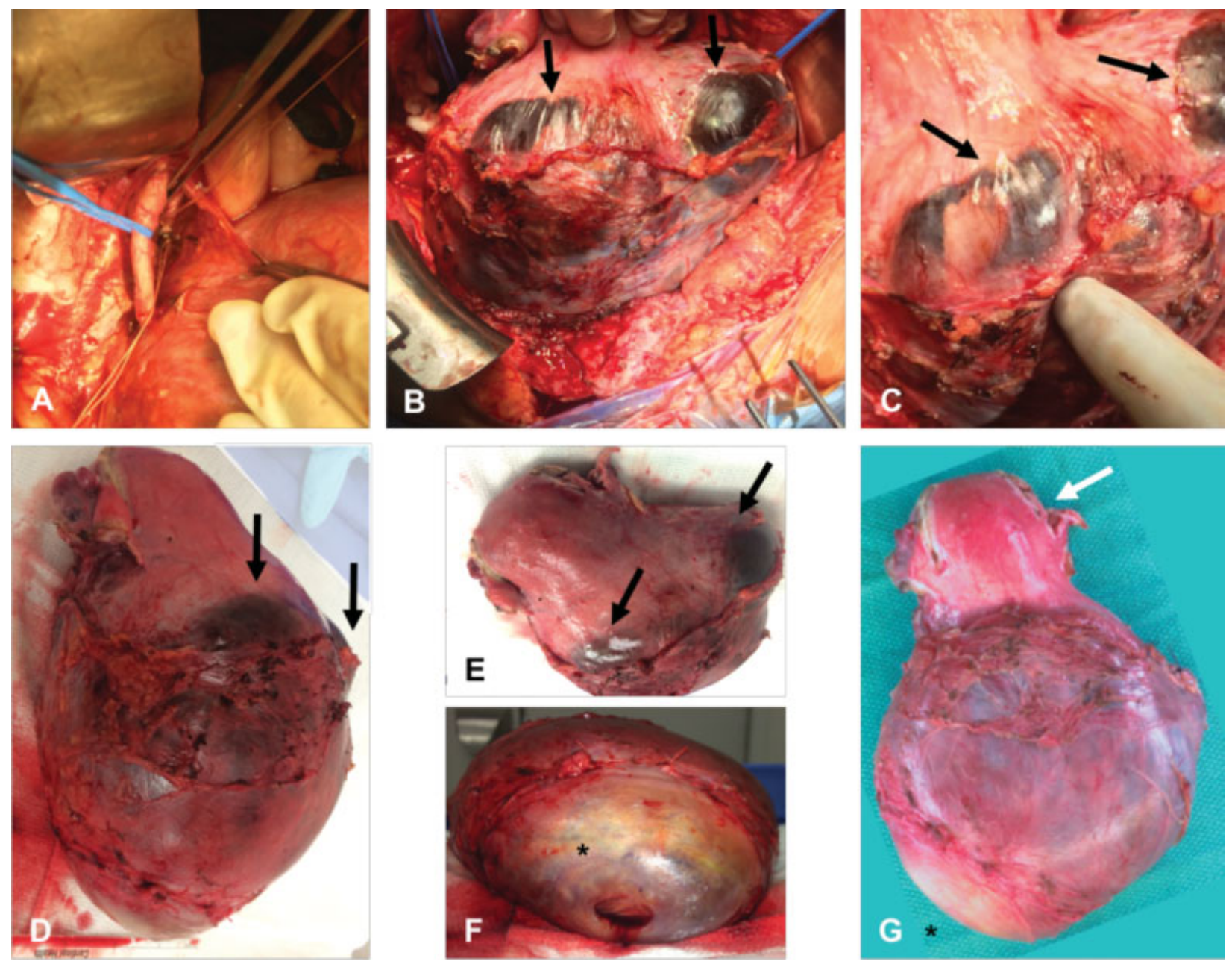

Fig. 2 Intraoperative specimen and gross specimen. Panel A: result of meticulous dissection and ureterolysis, prior to ligation of the anterior division of the internal iliac artery. Evident bulge of lower uterine segment (LUS) toward right pelvic sidewall. Right ureter indicated by blue tie. Right anterior internal iliac artery tied by the two sutures. Panel B: anterior surface of the LUS after dissection of fibrous adhesions. Black arrows indicate large areas of myometrial dehiscence with intact uterine serosa/amniotic membrane (uterine windows) that were concerning for impending uterine rupture. Panel C: fetal foot visible through single layer of uterine serosa/amniotic membrane (uterine window) indicated by black arrows. Panel D (right lateral view) and Panel E (left superior view): areas of myometrial dehiscence with intact serosa/amniotic membrane indicated by black arrows. Clear bulging of weakened left side of the LUS. Remnants of fibrous adhesions to the posterior bladder are evident, as well as abnormal vasculature overlying the anterior uterine wall. Panel F: widened cervical region $\left({ }^{*}\right)$ that is thinned and transparent, permitting visualization of placental vasculature. Dilated cervical os. Thinned cervix, cervical implantation, and cervical placenta percreta confirmed on histology. Panel G: anterior view of the uterus, rotated to align with the magnetic resonance imaging orientation of the uterus in - Fig. 1, Panel C. The fundus (white arrow) appears distinct from the LUS. The LUS is markedly widened and clearly hypervascular, grossly consistent with placenta percreta. Placental tissue distorts and stretches the LUS toward the patient's left. The cervical region $\left({ }^{*}\right)$ is thinned, markedly widened, and angled toward the patient's right. 
( - Fig. 1, Panel C, - Fig. 2, Panel G). Given these intraoperative findings, cesarean delivery was not performed prior to hysterectomy as the fetus was previable, and in the setting of vaginal bleeding, the risks of additional bleeding and delay associated with creation of a hysterotomy outweighed the potential benefits of delivery.

The surgical approach to the right uterine artery and right ureter was obscured by (1) the rightward displacement of the lower uterine segment containing extremely vascular placental tissue and (2) the fibrous adhesions between the bladder and the anterior uterus, which encased the ureter and right uterine artery. This required careful separation of the uterus from the right pelvic sidewall, meticulous right ureterolysis, and, ultimately, ligation of the anterior division of the right internal iliac artery ( - Fig. 2, Panel A). Due to the thick fibrous adhesions between the bladder and the uterus, an intentional $1-\mathrm{cm}$ cystotomy was performed in the bladder dome, which was repaired intraoperatively with a double-layer closure. The bladder was instilled with a mixture of sterile saline and sterile milk, with no discernable leakage. A Foley catheter was left to keep the bladder empty to promote healing. Estimated blood loss during surgery was $600 \mathrm{~mL}$; clinical stability and laboratory studies did not indicate any need for intraoperative transfusion.

On postoperative day 1 , the patient required two units packed red blood cells due to a drop in $\mathrm{Hgb}$ to $6.6 \mathrm{~g} / \mathrm{dL}$, which we suspected was due to a combination of hemodilution and modest underestimation of preoperative blood loss. The patient responded appropriately to transfusion and had no signs of ongoing bleeding. She was discharged on postoperative day 4 with a Foley catheter and leg bag, which was removed in the outpatient setting on postoperative day 7 after voiding cystourethrogram confirmed bladder integrity.
Pathological gross evaluation of the specimen confirmed significant distortion of the lower uterine segment to $14 \mathrm{~cm}$ and distortion of the cervical region to $7.5 \mathrm{~cm}$ ( - Fig. 2, Panels F and $\mathrm{G}$ ), as well as confirmed the attachment of the placental disc to the ectocervix, endocervix, and lower uterine segment anteriorly with minimal attachment posteriorly. There was noted to be marked hemorrhage throughout the placental parenchyma, with approximately $55 \%$ of the placental parenchyma replaced by clotted maternal blood, indicating likely placental abruption.

Microscopic examination confirmed implantation in the endocervix, with extensive placenta increta and focal placenta percreta (-Fig. 3), as well as a large acute retroplacental hematoma. The proximal endocervix was extremely thin with focal necrosis. The site of implantation in the endocervix contained only connective tissue and invasive trophoblasts without intervening myometrium or decidualized endometrium, establishing the diagnosis of placenta percreta (-Fig. 3 , Panel C). Other implantation sites in the lower uterine segment contain thinned myometrium with invasive placental trophoblasts and no intervening layer of decidualized endometrium, confirming placenta increta ( - Fig. 3, Panel B). The anterior wall of the uterus contained fibrous adhesions as well as bundles of smooth muscles that were presumed to be from the bladder. Identifiable endometrium was present in the fundus only and was partially covered by fibrinoid material with submucosal hyalinization, consistent with a history of endometrial ablation. Unlike the lower uterine segment, the fundus contained appropriately thick myometrium.

\section{Discussion}

This pregnancy with placenta percreta and incomplete uterine rupture after endometrial ablation significantly increased
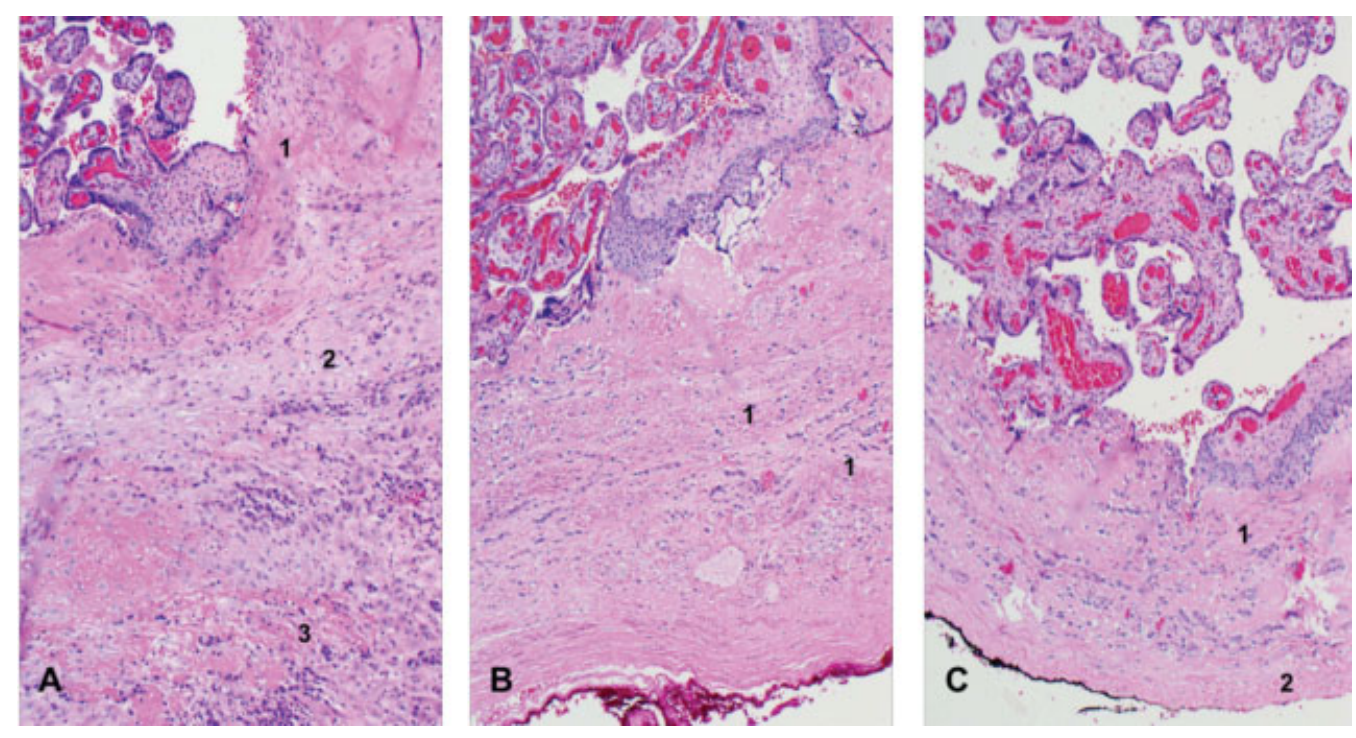

Fig. 3 Histologic evaluation of specimen. Panel A: normal decidualized endometrium. 1, Nitabuch's fibrinoid layer. 2, Decidualized endometrium with invasive trophoblasts. Also contains lymphocytes indicating abnormal, chronic inflammation, often seen with cervical implantation. 3 , Smooth muscle bundles of myometrium. Panel B: placenta increta. 1, Invasive trophoblasts in smooth muscle bundles, without a layer of decidualized endometrium. Thin layer of myometrium. Panel C: placenta percreta. 1, Invading trophoblasts among connective tissue, without decidualized endometrium or smooth muscle bundles of myometrium. 2, Uterine wall layer (not myometrium nor true serosa, as this was in a section of uterus inferior to the reflection of parietal peritoneum along the bladder). All panels viewed at $10 \times$ magnification. 
the patient's morbidity. MAP and its concomitant risk of hemorrhage are so much more prevalent in pregnancies following endometrial ablation and pose such serious risks that it has been suggested that postablation pregnancies should be assumed to have an adherent placenta until proven otherwise. ${ }^{12}$ These risks should be discussed with patients to ensure appropriate timing of ablation with regard to family planning, informed consent regarding continuation or termination of a pregnancy following endometrial ablation, and to allow appropriate delivery planning and preparation should the pregnancy continue. The complex surgical approach to placenta percreta described here underscores the importance of accurate antenatal diagnosis and surgical management in a practice setting with the multidisciplinary experience and capacity to manage such risks, particularly in a center of excellence. $^{13,14}$

This case is unique in that conception occurred either just before or just after endometrial ablation and concomitant bilateral tubal sterilization. Pregnancy occurring with or after tubal sterilization and/or endometrial ablation, though improbable, is not impossible. Two previous reports describe similar cases. Lo and Pickersgill described a pregnancy in which conception likely occurred a few weeks prior to endometrial ablation; the patient was not given a urine pregnancy test prior to the procedure. ${ }^{15}$ The pregnancy continued despite the ablation, but the patient elected to terminate the pregnancy at 12 weeks. El-Toukhy and Hefni described an unintended pregnancy that occurred 5 years after bilateral tubal ligation and 16 months after thermal balloon ablation; this pregnancy ended in a missed abortion at 10 weeks. ${ }^{16}$ We suspect that the morbidity encountered in our case was greater, in part, due to the more advanced gestational age at which the patient presented.

Both the manufacturer of NovaSure (Hologic Inc.) and the American College of Obstetricians and Gynecologists (ACOG) state that pregnancy after endometrial ablation can be dangerous for women and fetuses and that "appropriate contraception" should be used in women who could become pregnant. ${ }^{17,18}$ However, we could find no clear guidelines specifying the duration of use prior to ablation. Rather, pregnancy is listed as a contraindication to ablation. Our patient reported use of oral contraceptives prior to ablation as part of medical management of menorrhagia, had a negative pregnancy test prior to ablation, and underwent a tubal occlusion procedure at the time of ablation, arguably all of which constitute "appropriate contraception," as recommended by Hologic and ACOG. ${ }^{17,18}$ The makers of NovaSure recommend abstinence for at least 4 weeks postprocedure ${ }^{19}$; however, we found at least one medical Web site accessible to patients that suggests sexual activity may be resumed as early as 2 to 4 days postprocedure. ${ }^{20}$ The most recent ACOG Practice Bulletin does not address postprocedure recommendations for patient activity or follow-up. ${ }^{17}$ Contraceptive failure is well documented even with ideal use, among all forms of contraception, ${ }^{21,22}$ and bleeding patterns after ablation may not normalize for 3 months postprocedure, ${ }^{23}$ both of which likely contributed to the delay in pregnancy diagnosis in our patient.
This case generates the question of appropriate pregnancy testing in a woman with low likelihood of pregnancy but with significant risk factors for a highly morbid pregnancy. While the sensitivity for urine pregnancy testing nears $100 \%$ for normally implanted pregnancies, ${ }^{24-26}$ especially after 4 to 6 weeks of gestation, there still exists a small possibility for false-negative results. Quantitative serum pregnancy testing may have correctly identified this patient as pregnant, despite the negative qualitative urine test, and cost and time differences between the two tests are negligible. Providers should have a high index of suspicion for pregnancy in any women who has undergone ablation and who could possibly be pregnant, ${ }^{12,15,16}$ especially given the variability of bleeding after ablation, the imperfect use of contraception, and the frequency of adverse outcomes in postablation pregnancies. Quantitative serum pregnancy testing, or repeat qualitative urine pregnancy testing, may be merited in women who are about to undergo endometrial ablation or who have a history of ablation and subsequent indications of possible pregnancy.

In the United States, more than 300,000 endometrial ablations are performed annually, ${ }^{27}$ and more than 2.5 million women have undergone ablation with NovaSure alone. ${ }^{28}$ Endometrial ablation can substantially improve a woman's quality of life by reducing or eliminating menorrhagia. However, it is imperative that women undergoing endometrial ablation are counseled thoroughly about the serious risks of subsequent pregnancy and about the critical importance of effective contraceptive use after the procedure, even older women, those with postablation amenorrhea, and those with a history of infertility or tubal sterilization. The most recent review documented 123 pregnancies after endometrial resection or ablation, the vast majority of which were unintended and were associated with poor outcomes, including spontaneous abortion (28\%), ectopic pregnancy (6.5\%), preterm delivery (31\%), perinatal death $(14 \%)$, and one maternal death. ${ }^{11}$ Women who have undergone ablation should be tested for pregnancy should any signs or symptoms of pregnancy occur, given the physical and psychological risks associated with MAP.

\section{Disclosures \\ None.}

\section{Acknowledgments}

None.

\section{References}

1 Vahanian SA, Lavery JA, Ananth CV, Vintzileos A. Placental implantation abnormalities and risk of preterm delivery: a systematic review and metaanalysis. Am J Obstet Gynecol 2015;213(4, Suppl):S78-S90

2 Eshkoli T, Weintraub AY, Sergienko R, Sheiner E. Placenta accreta: risk factors, perinatal outcomes, and consequences for subsequent births. Am J Obstet Gynecol 2013;208(3):219.e1-219.e7

3 Belfort MA; Publications Committee, Society for Maternal-Fetal Medicine. Placenta accreta. Am J Obstet Gynecol 2010;203(5): 430-439 
4 Shawki O, Wahba A, Magon N. Abnormal uterine bleeding in midlife: the role of levonorgestrel intrauterine system. J Midlife Health 2013;4(1):36-39

5 Committee on Practice Bulletins-Gynecology. Practice bulletin no. 128: diagnosis of abnormal uterine bleeding in reproductiveaged women. Obstet Gynecol 2012;120(1):197-206

6 Miller JD, Lenhart GM, Bonafede MM, Basinski CM, Lukes AS, Troeger KA. Cost effectiveness of endometrial ablation with the NovaSure $\left({ }^{\circledR}\right)$ system versus other global ablation modalities and hysterectomy for treatment of abnormal uterine bleeding: US commercial and Medicaid payer perspectives. Int J Womens Health 2015;7:59-73

7 Pugh CP, Crane JM, Hogan TG. Successful intrauterine pregnancy after endometrial ablation. J Am Assoc Gynecol Laparosc 2000; 7(3):391-394

8 Xia E, Li TC, Yu D, et al. The occurrence and outcome of 39 pregnancies after 1621 cases of transcervical resection of endometrium. Hum Reprod 2006;21(12):3282-3286

9 Gervaise A, de Tayrac R, Fernandez H. Contraceptive information after endometrial ablation. Fertil Steril 2005;84(6):1746-1747

10 Daniels JP. The long-term outcomes of endometrial ablation in the treatment of heavy menstrual bleeding. Curr Opin Obstet Gynecol 2013;25(4):320-326

11 Yin CS. Pregnancy after hysteroscopic endometrial ablation without endometrial preparation: a report of five cases and a literature review. Taiwan J Obstet Gynecol 2010;49(3):311-319

12 Patni S, ElGarib AM, Majd HS, Edwards GJ, Ashraf MA. Endometrial resection mandates reliable contraception thereafter - a case report of placenta increta following endometrial ablation. Eur J Contracept Reprod Health Care 2008;13(2):208-211

13 Fox K, Shamshirsaz A, Belfort M. Center of Excellence for Placenta Accreta. In: Silver RM, ed. Placenta Accreta Spectrum. CRC Press; 2016. Chapter 9. In press

14 Silver RM, Fox KA, Barton JR, et al. Center of excellence for placenta accreta. Am J Obstet Gynecol 2015;212(5):561-568

15 Lo JS, Pickersgill A. Pregnancy after endometrial ablation: English literature review and case report. J Minim Invasive Gynecol 2006; 13(2):88-91

16 El-Toukhy T, Hefni M. Pregnancy after hydrothermal endometrial ablation and laparoscopic sterilisation. Eur J Obstet Gynecol Reprod Biol 2003;106(2):222-224
17 ACOG Committee on Practice Bulletins. ACOG Practice Bulletin. Clinical management guidelines for obstetriciangynecologists. Number 81, May 2007. Obstet Gynecol 2007;109(5):1233-1248

18 Hologic Inc. Novasure ${ }^{\circledR}$ Endometrial Ablation \& Birth Control. MISC-03509-001; 2015. Available at http://www.novasure.com/ sites/novasure/files/NovaSure_and_Birth_Control_0.pdf. Accessed September 1, 2016

19 Hologic Inc. Novasure ${ }^{\circledR}$ : What to Expect. 2016. Available at http:// www.novasure.com/patients/what-expect-novasure-procedure. Accessed September 1, 2016

20 Johns Hopkins Health Library. Endometrial ablation. Available at http://www.hopkinsmedicine.org/healthlibrary/test_procedures/gynecology/endometrial_ablation_92,p07774/. Accessed September 1, 2016

21 Polis CB, Bradley SE, Bankole A, Onda T, Croft T, Singh S. Typical-use contraceptive failure rates in 43 countries with Demographic and Health Survey data: summary of a detailed report. Contraception 2016;94(1):11-17

22 Centers for Disease Control and Prevention. Effectiveness of Family Planning Methods. Infographic. 2014. Available at http://www. cdc.gov/reproductivehealth/unintendedpregnancy/pdf/familyplanning-methods-2014.pdf. Accessed September 1, 2016

23 Hologic Inc. NovaSure ${ }^{\circledR}$ Endometrial Ablation. Summary of Risks and Considerations. MISC-01532-001; 2015. Available at http:// www.novasure.com/sites/novasure/files/NovaSure_Patient_Risks-and-Considerations.pdf. Accessed September 1, 2016

24 Fromm C, Likourezos A, Haines L, Khan AN, Williams J, Berezow J. Substituting whole blood for urine in a bedside pregnancy test. J Emerg Med 2012;43(3):478-482

25 Mazouz S, Lee JK, Fernandez H. Evaluation of a urinary test as a diagnostic tool of a nonprogressive pregnancy. Fertil Steril 2011; 95(2):783-786

26 Wilcox AJ, Baird DD, Dunson D, McChesney R, Weinberg CR. Natural limits of pregnancy testing in relation to the expected menstrual period. JAMA 2001;286(14):1759-1761

27 Hologic Inc. One Million Patients Choose Hologic's NovaSure ${ }^{\circledR}$ System Over Hysterectomy. 2009. Available at http://investors. hologic.com/news?item=420. Accessed September 1, 2016

28 Hologic Inc. Personal Communication with manufacturer. Data on file. Inquiry made September 22, 2016 